\title{
Análisis de la Gestión del Mantenimiento orientado a infraestructuras para el desplazamiento de discapacitados en el Complejo Hotelero los Cactus Tuxpan, Varadero.
}

\author{
Analysis of Maintenance Management oriented to infrastructures for the \\ displacement of the disabled in the Los Cactus Tuxpan Hotel Complex, \\ Varadero.
}

Naylet Sangroni Laguardia. ${ }^{1}$, Jessie Arlene Pérez Castañeira. ${ }^{2}$, Roxanna Alba Cruz. ${ }^{3}$, Bisleivys Jiménez Valero. ${ }^{4}$ \& Luis Efraín Velastegui López. ${ }^{5}$

\begin{abstract}
.
DOI: https://doi.org/10.33262/concienciadigital.v4i2.1.1716

Introduction. Tourism is one of the main dynamizing activities of the world economy, currently accessible tourism is an opportunity aimed at raising awareness of the tourism industry with services that meet the requirements for adequate care for people with disabilities, where the application of coherent Industrial Maintenance Policies contributes to achieving the standards desired by this market segment. Objective. Analyze the Maintenance Management oriented to infrastructures for the displacement of the disabled in the Los Cactus Tuxpan Hotel Complex, Varadero. Methodology. Different theoretical scientific methods were applied: analytical-synthetic, inductive-deductive, systemicstructural, bibliometric, statistical (correlation), review of the state of the art of accessible tourism, care for the disabled and maintenance of infrastructures through exploratory

\footnotetext{
${ }^{1}$ Estudiante. Universidad de Matanzas, Sede Camilo Cienfuegos, Autopista a Varadero, km 3 1⁄2, Matanzas, Cuba. Email: naylet.sangroni@ nauta.cu;

${ }^{2}$ Estudiante. Universidad de Matanzas, Sede Camilo Cienfuegos, Autopista a Varadero, km 3 1⁄2, Matanzas, Cuba. Email: jessiarlene@ nauta.cu

${ }^{3}$ Estudiante. Universidad de Matanzas, Sede Camilo Cienfuegos, Autopista a Varadero, km 3 1/2, Matanzas, Cuba. Email: roxanna.alba.cruz@gmail.com

${ }^{4}$ Doctora en Ciencia. Universidad de Matanzas, Matanzas, Cuba. Email: bisleivys.jimenez@umcc.cu Orcid. http//orcid.org/0000--0003-4812-4558

${ }^{5}$ Ciencia Digital Editorial, Ecuador, luisefrainvelastegui@cienciadigital.org
} 
methodology -descriptive. Direct observation, brainstorming, expert method with the help of VOSviewer 1.6.4 software and tools such as: Microsoft Office, Visio 2010 and Project Professional 2010. Results were used. 1. A review of the state of the art of accessible tourism, care for the disabled and maintenance of infrastructures was carried out. 2. A map of the bibliometric study of the term "care for the disabled" was obtained, which determines the main lines of research on the subject. 3. A procedure was proposed for the analysis of the management of the maintenance of the infrastructures for the displacement of the disabled in the Los Cactus Tuxpan Hotel Complex, Varadero. 4. A corrective measures plan was proposed in order to eradicate the problems detected during the diagnosis in the entity under study. Conclusions. The importance and timeliness of the subject was systematized, and the need to apply the proposed procedure for the management of the maintenance of infrastructures for the displacement of the disabled in the Los Cactus Tuxpan Hotel Complex, Varadero, was highlighted.

Keywords: accessible tourism, disabled, maintenance, infrastructure.

\section{Resumen.}

Introducción. El turismo es una de las principales actividades dinamizadoras de la economía mundial, en la actualidad el turismo accesible supone ser una oportunidad encaminada a la sensibilización de la industria turística con servicios que cumplan con los requerimientos para la atención adecuada a personas con discapacidad, donde la aplicación de Políticas coherentes de Mantenimiento Industrial contribuye a alcanzar los estándares deseados por este segmento del mercado. Objetivo. Analizar la Gestión del Mantenimiento orientado a infraestructuras para el desplazamiento de discapacitados en el Complejo Hotelero los Cactus Tuxpan, Varadero. Metodología. Se aplicaron diferentes métodos científicos teóricos: analítico-sintético, inductivo-deductivo, sistémico-estructural, bibliométricos, estadísticos (correlación), revisión del estado del arte del turismo accesible, atención a discapacitados y mantenimiento de infraestructuras a través de la metodología exploratoria-descriptiva. Se utilizó la observación directa, tormenta de ideas, método de expertos con la ayuda del software VOSviewer 1.6.4 y herramientas como: Microsoft Office, Visio 2010 y Project Profesional 2010. Resultados. 1. Se realizó una revisión del estado del arte del turismo accesible, atención a discapacitados y mantenimiento de infraestructuras. 2. Se obtuvo un mapa del estudio bibliométrico del término "atención a discapacitados" que determina las principales líneas de investigación en el tema. 3. Se propuso un procedimiento para el análisis de la gestión del mantenimiento de las infraestructuras para el desplazamiento de discapacitados en el Complejo Hotelero los Cactus Tuxpan, Varadero. 4. Se propuso un plan de medidas correctivas con el fin de erradicar los problemas detectados durante el diagnóstico en la entidad objeto de estudio. Conclusiones. Se sistematizó la importancia y actualidad del tema, y quedó evidenciado la necesidad de aplicación del procedimiento propuesto para la gestión del mantenimiento de las infraestructuras para el desplazamiento de discapacitados en el Complejo Hotelero los Cactus Tuxpan, Varadero. 
Palabras claves: turismo accesible, discapacitados, mantenimiento, infraestructuras.

\section{Introducción.}

El turismo es una actividad que involucra a toda la sociedad, ya sea directa o indirectamente; y ha experimentado un auge en las últimas décadas, al presentar cifras que crecen de forma exponencial (Suárez Falcón, Verano Tacoronte, \& García Santana, 2016). La globalización de los mercados turísticos ha favorecido la diversificación de destinos y de los segmentos de consumidores. Por ello, el turismo se ha forjado hasta convertirse en un verdadero derecho fundamental, indispensable para el pleno desarrollo de la dignidad humana (Clemente Soler, Bote Díaz, \& Sánchez Vera, 2018). A medida que la sociedad se desarrolla, el estado del turismo cambia de ser socialmente deseable a ser una necesidad (Agovino, Casaccia, Garofalo, \& Marchesano, 2017). Existen casos donde estos propósitos no se cumplen debido a características ambientales, físicas y sociales que hacen de la experiencia turística incómoda, poco segura, y sin equidad (Cruz, 2019).

Uno de los segmentos de mercado que ha cobrado fuerza en los últimos años es el de las personas discapacitadas, que repercute en diferentes espacios de la economía, sociedad o política, entre otros, y se ha hecho evidente en el turismo (Carossia, Narvaez, \& Peluc, 2007). Se pueden considerar diferentes tipos de personas con discapacidad: aquellas que presentan una discapacidad intelectual, las de limitaciones motoras o físicas, y las que muestran una discapacidad auditiva o visual. Sin embargo, existen otras series de circunstancias que pueden ocasionar una restricción temporal de la movilidad, lo que reduce la independencia y la autonomía de las personas, en este sentido, se encuentran los adultos mayores, personas con obesidad, mujeres en gestación, personas que viajan con bebés o niños pequeños (Cruz, 2019).

Este segmento de mercado viene justificado por el número de personas que lo integran y su participación en el mercado turístico. Por tanto, el turismo accesible se ha planteado como un turismo para todos que incluye procesos colaborativos entre personas que brindan servicios, mejora a la infraestructura, considera las dimensiones de acceso, y brinda valores de igualdad y verdadera inclusión para todas las personas, aún si éstas presentan alguna discapacidad. (Kastenholz, Eusébio, Figueiredo, \& Lima, 2012). Por esta razón es importante conocer sobre el derecho al acceso y disfrute de espacios recreativos y los obstáculos intangibles que excluyen o incluyen a personas con discapacidad (ONU, 2014).

El término turismo accesible o turismo para todos es relativamente reciente, fue utilizado por primera vez en el Informe Baker publicado en el Reino Unido en 1989, y hace referencia a la cuestión de la accesibilidad a través de la supresión de barreras urbanísticas y arquitectónicas, así como a la integración social a través del turismo de las personas con algún tipo de inhabilidad (Jurado Almonte, 2014).

Las ventajas de promover la gestión del turismo accesible parte desde el punto de vista social, el cual incide en el incremento de la calidad de vida de las personas, desde la 
perspectiva del marketing donde contribuye a mejorar la imagen comercial de las organizaciones turísticas. En el ámbito económico resulta interesante, permite atraer a turistas y visitantes de atención prioritaria, entre ellos las personas con discapacidad que suelen viajar acompañados por otros clientes, razón por la cual son considerados como multiclientes (Tite Cunalata et al., 2021).

Para Rifaï, (2014) el turismo ha sido fuertemente potenciado en este último tiempo y esto ha generado la urgente necesidad de adaptar los espacios urbanos y las instalaciones de tal forma de satisfacer las necesidades y requerimientos de todos los turistas con discapacidad alguna que no le permita desplazarse adecuadamente. En consecuencia, Denisse Gómez Bañuelos, Pérez Gaxiola, \& Woolfolk Gallego, (2018) plantean que es importante conocer las necesidades y deseos de las personas discapacitadas en su comportamiento como turistas, para tratar de perfilar una oferta turística cuyo contenido esté adaptado a las mismas, a su vez que contribuya a dar una mayor satisfacción a los viajeros discapacitados y una mayor competitividad a las empresas turísticas.

A partir del aumento de las tasas de discapacidad, debido en parte al envejecimiento de la población y al aumento de la prevalencia de enfermedades crónicas, la infraestructura turística es la base tangible del desarrollo del turismo, dentro de esta se encuentran la red de comercialización, servicios de transportes, la prestación de servicios básicos, restaurantes, museos, hoteles, etc. Para atender esta demanda se necesita que se realicen adecuaciones en las instalaciones y servicios para facilitar el acceso y calidad de uso a este grupo, además de una formación continua, posibilitando el mejoramiento de las competencias y cualificaciones de los empleados. Por ello es necesario una adecuada gestión de mantenimiento en cada infraestructura para que cada recurso turístico brinde lo mejor a cada visitante y sea de disfrute de cada uno de ellos.

Bien sabemos que el mundo entero tiene derecho al descanso y disfrute de su tiempo de ocio. Pero muchas veces no se presentan las condiciones adecuadas para que esto suceda. Es por ello, que es importante destacar la posibilidad de llevar adelante una planificación turística que tenga en cuenta a todos sus actores, involucrándolos en su diversidad, para lograr promover la igualdad y la inclusión mediante un turismo accesible (Sanz Labrador, 2018).

En tal sentido, las empresas generadoras de bienes y/ o servicios que utilizan instalaciones, edificios, dispositivos, etc., para lograr su objetivo social y empresarial, necesitan que estos activos se mantengan en buen estado de funcionamiento, de confiabilidad, de mantenibilidad y de disponibilidad, acorde con sus necesidades, por lo cual las organizaciones empresariales deben procurar que la vida útil de sus activos físicos sea la máxima alcanzable al mínimo costo posible (Proaño Cárdenas, 2018). Por ello el mantenimiento, es actualmente reconocido como un elemento fundamental para incrementar la competitividad al propiciar la prolongación de la vida útil de los componentes y contribuir a mejorar la calidad de los productos. En las instalaciones hoteleras, el mantenimiento desempeña un papel fundamental para el desempeño creciente de la institución. Por esta razón la presente investigación tiene como objetivo: 
analizar la gestión del mantenimiento orientado a infraestructuras para el desplazamiento de discapacitados en el Complejo Hotelero los Cactus Tuxpan, Varadero.

\section{Metodología.}

En la presente investigación se aplicaron diferentes métodos científicos teóricos: analítico-sintético, inductivo-deductivo, sistémico-estructural, bibliométricos y estadísticos (correlación). Se realiza una revisión del estado del arte del turismo accesible, atención a discapacitados y mantenimiento de infraestructuras para el periodo comprendido entre 2002-2021 y para ello se emplea una metodología exploratoriadescriptiva, en la cual se parte de seleccionar palabras clave y plantear criterios de búsqueda normalizada y sistematizada de información.

Se confecciona un estudio bibliométrico de tipo cuantitativo-descriptivo de la producción científica a partir de la base de datos ScienceDirect (https://www.sciencedirect.com/), plataforma digital que permite la búsqueda parametrizadas en el grupo de campos: Title, abstractor keywords, con el objetivo de ver el vínculo entre palabras claves y temáticas. La estrategia de búsqueda empleada, que se restringe solo al período 2017- 2021, es: "desplazamientos de discapacitados" con $(\mathrm{N}=821)$. A partir de lo planteado en el título, resumen y palabras claves de las investigaciones, se determinó que solo 117 documentos estaban vinculados al objeto de estudio de la investigación. Posteriormente se procesaron cada uno de los archivos, y se utilizaron para visualizar y analizar las tendencias en forma de mapa bibliométrico.

Luego de consultar referentes bibliográficos en las temáticas de mantenimiento a infraestructuras para el desplazamiento de discapacitados en instituciones turísticas se tuvo en cuenta como marco metodológico el procedimiento de la figura 1 en aproximación a Borroto Pentón, (2005), con el fin de analizar, evaluar y proponer acciones correctivas para mejorar la gestión de mantenimiento de las infraestructuras para el desplazamiento de discapacitados en el Complejo Hotelero los Cactus Tuxpan, Varadero.

Etapa 1. Preparación

- Paso 1.1. Caracterización de la entidad objeto de estudio.

- Paso 1.2. Conformación de equipo de trabajo.

Etapa 2. Realización del diagnóstico de la situación existente en la gestión del mantenimiento en El Complejo Hotelero los Cactus Tuxpan.

- Paso 2.1. Preparación del plan de aspectos a diagnosticar.

- Paso 2.2. Asignación de tareas.

Etapa 3. Determinación de los principales problemas que afectan la gestión del mantenimiento en El Complejo Hotelero los Cactus Tuxpan.

- Paso 3.1. Problemas detectados en la gestión de mantenimiento.

Etapa 4. Propuestas de soluciones a los problemas detectados.

- Paso 4.1. Listado de soluciones.

- Paso 4.2. Propuesta de un plan de Mantenimiento.

Figura 1. Metodología utilizada para el análisis de la gestión de mantenimiento.

Fuente. Elaboración propia. 
El procedimiento consta de las siguientes etapas:

Etapa 1. Preparación. Esta etapa consiste en preparar el campo de estudio para la investigación, al determinar las características de la entidad objeto de estudio y la conformación del grupo de trabajo.

Paso 1.1. Caracterización de la entidad objeto de estudio: se definen las características de la entidad objeto de estudio en cuanto a datos generales y objetivos.

Paso 1.2. Conformación de equipo de trabajo: se crea el equipo de trabajo que tiene como función la aplicación del procedimiento general propuesto en la presente investigación. Para su conformación se tiene en cuenta que el trabajo con grupos de expertos debe estar avalado por su grado de experticidad, aspecto que ha sido destacado por varios autores (Artola Pimentel, 2002; Negrín Sosa, 2003). La experiencia, calificación y capacidad de los miembros del equipo que participarán en la investigación deben estar acreditadas por su grado de experticia. En el presente artículo se analiza el procedimiento propuesto por Artola Pimentel, (2002), en el que se destaca el cálculo del índice de experticidad (IE).

Etapa 2. Realización del diagnóstico de la situación existente en la gestión del mantenimiento en El Complejo Los Cactus Tuxpan: en este paso se realiza el diagnóstico del estado de la gestión del mantenimiento en la instalación con la preparación del plan de aspectos a diagnosticar y la asignación de recursos.

Paso 2.1. Preparación del plan de aspectos a diagnosticar: el jefe del equipo inspector prepara un plan de inspección que proporciona la base para el acuerdo entre todos los implicados.

Paso 2.2. Asignación de tareas: el jefe de la comisión asigna las áreas que resultan objeto de investigación a cada uno de los integrantes del comité con el fin de lograr los objetivos propuestos con la calidad requerida en el menor tiempo posible.

Etapa 3. Determinación de los principales problemas que afectan la gestión del mantenimiento en El Complejo Los Cactus Tuxpan. El equipo de inspección se reúne y mediante una tormenta de ideas decidieron los principales problemas que presenta la gestión del mantenimiento en las infraestructuras para el desplazamiento de personas con discapacidad en el Complejo Hotelero los Cactus Tuxpan.

Paso 3.1. Problemas detectados en la gestión de mantenimiento: se listaron los problemas detectados.

Etapa 4. Propuestas de soluciones a los problemas detectados: se proponen un grupo de medidas correctivas encaminadas a dar solución a los problemas detectados.

Paso 4. 1. Listado de soluciones: se listan las soluciones propuestas por el grupo de trabajo.

Paso 4.2. Propuesta de un plan de Mantenimiento. Debido a la necesidad de la programación oportuna, la existencia de la documentación necesaria de las misma y el número de quejas registrados durante los dos meses que se desarrolla la inspección por el 
mal estado técnico de las infraestructuras orientadas al desplazamiento de personas discapacitadas, se aplica el Método Pareto y se propuso un plan de mantenimiento preventivo.

Así mismo, para el análisis de la información se emplea el software VOSviewer 1.6.4 y herramientas como Microsoft Office, Visio 2010, Project Profesional 2010.

\section{Resultados.}

El turismo accesible se origina en Europa en la década de los ochenta del siglo xx, específicamente en los países de Alemania y el Reino Unido, que en materia de accesibilidad lograron el mayor avance; luego, este tipo de turismo llega a América, donde su progreso se manifiesta en particular en Argentina con el programa "Para un Turismo Accesible a los Minusválidos", promovido en los años noventa en Buenos Aires (Fraiz Brea, Alén González, \& Domínguez Vila, 2008)). Esta nueva concepción del turismo, además de contribuir a una mayor especialización de las instalaciones que lo desarrollan, permite mostrar un aspecto humanístico que mejora la imagen corporativa de la empresa turística socialmente responsable, lo que se traduce en una importante ventaja competitiva (Kastenholz, 2009). Su base de evolución se centra en la accesibilidad universal, los tipos de discapacidad, los mercados turísticos y la legislación que ampara los derechos de las personas con discapacidad (Martínez Carrillo \& Boujrouf, 2020).

Según Castillo García, (2019) el concepto de accesibilidad se ha abierto camino más allá de la eliminación de barreras físicas, y hoy considera a la persona y a su entorno como un todo. Es por ello que se han desarrollado a partir de él, diversos enfoques cuyo alcance va más allá de las respuestas que ofrecen las normativas. Por tales motivos la accesibilidad debe ser considerada un bien público, consagrada en términos de derecho ciudadano, a través del cual toda persona, sin importar su edad, genero, religión, condición física, tiene derecho a interactuar socialmente y disfrutar, con autonomía, de todos los servicios que proporciona la comunidad.

En la Tabla No 1 se muestran de las principales definiciones encontradas sobre turismo accesible comprendido en los años 2017 hasta la actualidad.

\begin{tabular}{ll}
\hline Autor/Año & Definición \\
\hline (Castro, 2017) & $\begin{array}{l}\text { Turismo inclusivo es el conjunto de actividades realizadas durante el } \\
\text { tiempo libre dedicado al turismo por personas con discapacidad, que les } \\
\text { posibilita su plena integración desde la óptica funcional y psicológica, y les } \\
\text { permite la plena satisfacción individual y social. }\end{array}$ \\
(Miranda, 2017) & $\begin{array}{l}\text { Turismo accesible, el cual pueda ser utilizado por todo tipo de persona y } \\
\text { que alude a cualquier habilidad o capacidad que esta posea; es lo que se } \\
\text { pretende hacer al adaptar los deportes extremos, buscar que todas las } \\
\text { personas puedan disfrutar de ellos de manera segura y confiable. }\end{array}$ \\
(Guerrero, 2018) & $\begin{array}{l}\text { Permite a todas las personas participar en las actividades sociales y } \\
\text { económicas para las que se ha concebido el entorno construido. }\end{array}$ \\
(Barba, 2018) & $\begin{array}{l}\text { El turismo accesible garantiza el uso y disfrute del turismo, } \\
\text { independientemente de las capacidades, del estado o de la condición de } \\
\text { las personas. }\end{array}$ \\
\hline
\end{tabular}


(Nonome, 2019) Es el proceso de comprensión de los elementos de empuje en la experiencia del turista con necesidades de acceso.

(Tite, Carrillo, \& El turismo accesible es un área del conocimiento que estudia la integración Ochoa, 2021) de dimensiones e indicadores que están incorporados de forma transversal a los procesos en las organizaciones para permitir innovar sus productos turísticos de manera tal que satisfagan a todos los segmentos, incluidos los turistas y visitantes de atención prioritaria, sin olvidar la rentabilidad de este tipo de actividad.

\section{Fuente: Elaboración propia.}

Tabla No 1. Diferentes definiciones sobre turismo accesible.

En tal sentido, el turismo accesible alcanza cada vez más excelencia en los diferentes países del mundo. En el proceso de evolución del turismo accesible se observa la tendencia creciente de esta modalidad en países latinoamericanos como México, Argentina, Chile, Colombia y Ecuador, que han mostrado mayor interés en generar una oferta innovadora con respecto a las necesidades de turistas y visitantes de atención prioritaria. También se evidencia el desarrollo en América Latina de políticas públicas que establecen la accesibilidad universal, así como el derecho al deporte, al ocio, al turismo, a la salud, a la educación, etc. Todo lo anterior contribuye a asegurar la plena participación de este tipo de clientes y el goce de sus derechos, entre ellos, el derecho al turismo en igualdad de condiciones (Tite et al., 2021).

En el contexto internacional se han desarrollado conferencias que abordan el tema de turismo accesible, entre las que se puede mencionar, la Asamblea General celebrada en Manila, que recoge el derecho al turismo de las personas discapacitadas con las mejores condiciones de acceso y sin discriminación. En este sentido muchos destinos internacionales han desarrollado obras encaminadas a mejorar la accesibilidad turística (Castro, 2017): se han creado rampas en los museos y sitios de interés en países como Austria, España y Ecuador, que además cuentan con calles peatonales para una mejor movilidad (Pinotti, 2018). Las playas inclusivas con parqueos accesibles para todos, rampas, señalética, equipamiento, pasarelas (Vergara Peña, 2016) son un modelo que ya existe en países como Estados Unidos (Charles Mears State Park, en Michigan), España (Arona, en Tenerife, así como en Cataluña y Valencia), Francia (Nimes, en Gard), Perú (Punta Negra y Miraflores, en Lima), Chile (Frutillar, en la Región de Los Lagos), México (Cuastecomates en Jalisco, Bahía de Kino en Sonora, Playa Delfines en Quintana Roo y Playa bonita en Campeche), entre otras (D. Gómez Bañuelos, Perez Gaxiola, \& Woolfolk Gallego, 2018).

En Cuba, después del triunfo revolucionario, gran parte de las inversiones se destinaron al desarrollo y expansión de importantes obras de infraestructura de apoyo al turismo (Silveira, Silveira, \& Castellanos, 2010). En el polo turístico de Varadero se realizan constantemente obras de restauración como solución de accesibilidad hacia y desde el polo para canalizar y distribuir el aumento esperado del número de turistas. En algunos hoteles hay rampas para facilitar el acceso de personas discapacitadas, en restaurantes y bares hay espacios para sillas de ruedas, se han creado paseos peatonales costeros y de playa como enlace entre las zonas urbanizadas y naturales con un acentuado carácter 
peatonal, con rampas y escaleras, pasarelas, vayas, aparcamientos y señalización (Alonso Gatell \& Leyva Fontes, 2008).

Sanz Labrador, (2018) plantea que un turismo que trascienda, y que tenga como objetivo principal crear espacios turísticos integradores, es decir, desarrollados para que cada persona pueda disfrutarlos, bajo las mismas condiciones sin distinción alguna, es lo que se necesita hoy en día, dejando de lado las diferencias y aceptando la diversidad. Las personas con discapacidad deben tener los mismos derechos y brindar iguales oportunidades que a todos los demás seres humanos, enfatizando que los diversos países deben incluir a la población discapacitada en el sector turístico y demás actividades, con oportunidades similares para participar en actividades recreativas en viajes, hoteles, organizaciones voluntarias y otros, con el fin de garantizarles un mejor servicio a todas las personas mientras tengan las capacidades intelectuales para su disfrute (Castro-Alfaro, Paz-Marcano, \& Pinto-Aragón, 2019).

La existencia de diferencias individuales es un hecho inherente a la condición humana. Las características personales que nos identifican como seres únicos e irrepetibles imponen retos a todos los sectores de la sociedad. Esto requiere de cambios en la forma de interactuar, pensar, comprender y ofrecer oportunidades a la diversidad de las necesidades crecientes y a las diferencias de contextos teniendo como una alternativa una inclusión de calidad (Sanz Labrador, 2018). El logro de la integración a la sociedad de todas las personas con necesidades asociadas o no a las discapacidades en contextos cada vez más inclusivos, depende de la concientización, sensibilización y el cambio de todos los miembros de la comunidad en su conjunto (Nonome, 2019).

Según Domínguez, Fraiz, \& Alén, (2016) existe un incremento de personas con discapacidad que viajan, sobre todo en las últimas dos décadas, pero existen muchas las razones para que este suceso haya ocurrido. Entre las causas que han llevado a este incremento de turismo/viajes de personas con discapacidad debemos destacar, fundamentalmente, su gran disponibilidad de tiempo libre y sus grandes deseos de viajar, potenciados estos últimos por la existencia de un mayor número de ayudas técnicas y económicas, todo lo cual ha aumentado las posibilidades de las personas discapacitadas a la hora de materializar sus deseos de viajar.

Para Sanz Labrador, (2018) el turismo, como parte de la sociedad está impuesto a lograr este reto, pero para ello es necesario eliminar una serie de barreras que en lo físico y en lo personal impiden el completo acceso de las personas con discapacidad a las instalaciones turísticas. Bien sabemos que el mundo entero tiene derecho al descanso y disfrute de su tiempo de ocio. Pero muchas veces no se presentan las condiciones adecuadas para que esto suceda. Es por ello, que es importante destacar la posibilidad de llevar adelante una planificación turística que tenga en cuenta a todos sus actores, involucrándolos en su diversidad, para lograr promover la igualdad y la inclusión mediante un turismo accesible. 
En correspondencia se desarrolla un mapa del estudio bibliométrico del término "desplazamientos de discapacitados" en base a la co-ocurrencia de palabras (Tabla 2), con el uso del software VOSviewer 1.6.4.

Tabla 2. Mapa del estudio bibliométrico.

\section{Desplazamiento de discapacitados}

\section{Atributos de mayor co-ocurrencia}

Calidad de vida, envejecimiento, dependencia, lucha por independencia, vejez, vulnerabilidad, actividad física, desplazamientos, infraestructuras adecuadas, lugares accesibles, caídas, sillas de ruedas, accidentabilidad, prevención, movilidad.

\begin{tabular}{lll}
\hline Año & Ítems & Clúster \\
\hline $2016-2021$ & 51 & 7 \\
\hline
\end{tabular}

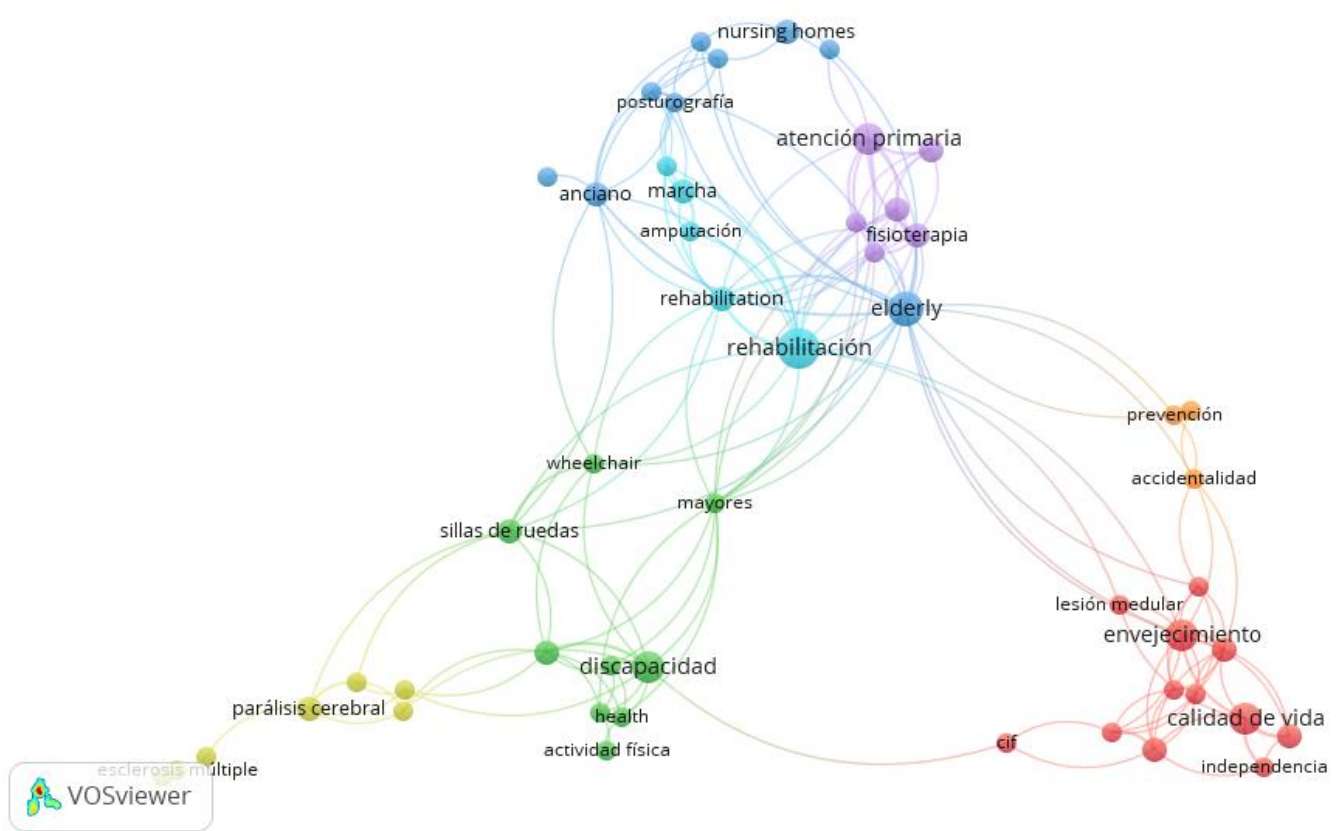

Fuente: Elaboración propia.

Los resultados obtenidos demuestran que las infraestructuras adecuadas, lugares accesibles y los desplazamientos para personas discapacitadas constituyen referentes de estudios a nivel mundial según la base de datos Sciencie Direct. De acuerdo con los resultados obtenidos Domínguez Vila, Fraiz Brea, \& Alén González, (2011) plantean que la discapacidad más común es la que implica reducción de la movilidad entre ellas se encuentran las personas cuya discapacidad es permanente o tiene probabilidades de aumentar y son las que utilizan silla de ruedas (SR), caminadores, muletas, bastones u otras tecnologías para desplazarse. Por otro lado, están las que tienen discapacidades temporales es decir que su estado funcional tiende a mejorar, por ejemplo, las personas enyesadas o las mujeres gestantes.

El mantenimiento de las infraestructuras orientadas para discapacitados en las instalaciones hoteleras contribuye a lograr que cualquier individuo puede recrearse o 
hacer uso de los servicios en cualquier instalación sin importar su condición física. Se reconoce que el mantenimiento es todo el trabajo necesario para mantener en buen estado de funcionamiento todo tipo de bienes, como las instalaciones y equipos en condiciones de funcionamiento seguro, eficiente y económico (Mercado Britton \& Villorina Seña, 2010). La gestión del mantenimiento no es un proceso aislado, sino que es un sistema linealmente dependiente de factores propiamente ligados a la gestión del mantenimiento, así como de factores internos y externos a la organización (González Echavarría, Martínez Delgado, Barreto San Germán, Espinosa Alfonso, \& Cabrera Gómez, 2020).

La actividad de mantenimiento, independientemente de la entidad en que se desarrolle, debe lograr la reducción de las averías imprevistas y del tiempo de reparación de los activos fijos, debe procurar la prolongación de la vida útil de los componentes, con el correspondiente ahorro de recursos y energía y con ello reducir el costo de mantenimiento de las instalaciones, para dar como resultado la mejora continua de la calidad y la eficiencia de los servicios (Suárez Fragas, Medina Peña, \& Hernández Alfonso, 2016).

Los objetivos del mantenimiento según (Ayala, 2018) son:

$\checkmark$ Mejorar la disponibilidad de las instalaciones.

$\checkmark$ Mejorar la fiabilidad y la calidad del servicio

$\checkmark$ Evitar, reducir, y en su caso, reparar, las fallas.

$\checkmark$ Disminuir la gravedad de las fallas que no se lleguen a evitar.

$\checkmark$ Evitar detenciones inútiles o paro de máquinas.

$\checkmark$ Evitar accidentes.

$\checkmark$ Maximizar la productividad y eficiencia.

$\checkmark$ Alcanzar o prolongar la vida útil de equipos e instalaciones.

$\checkmark$ Aumentar la seguridad de personas, equipos e instalaciones.

$\checkmark$ Reducir costos.

Las funciones del Mantenimiento propuestas por autores como: (Borroto Pentón, 2005; Jiménez-Castro, 2017) se ilustran en la tabla No 3.

Tabla 3. Funciones del Mantenimiento.

\begin{tabular}{rll}
\hline Funciones & \multicolumn{1}{c}{ Caracterización } \\
\hline Primarias & $\checkmark \quad$ Mantener, reparar y revisar los equipos e \\
& instalaciones. \\
& $\checkmark \quad$ Generación y distribución de los servicios \\
& eléctricos, vapor, aire, agua, gas, etc. \\
$\checkmark$ & Modificar, instalar, remover equipos y \\
& edificios. \\
$\checkmark$ & Nuevas instalaciones de equipos y \\
& edificios. \\
$\checkmark$ & Desarrollo de programas \\
& mantenimiento preventivo y programado. \\
$\checkmark$ & Selección y entrenamiento de personal. \\
$\checkmark$ & Asesorar la compra de nuevos equipos. \\
$\checkmark$ & Hacer pedidos de repuestos, \\
Secundarias & herramientas y suministros.
\end{tabular}


$\checkmark$ Controlar y asegurar un inventario de repuestos y suministros.

$\checkmark$ Mantener los equipos de seguridad y demás sistemas de protección.

$\checkmark \quad$ Llevar la contabilidad e inventario de los equipos.

$\checkmark$ Cualquier otro servicio delegado por la administración.

Fuente: Elaboración propia.

El mantenimiento en la actualidad se puede clasificar, conforme a las actividades y planes de acción realizadas en la industria, también considerados como labores, etapas o generaciones del mantenimiento (Tabla No 4) (Buitrago Falcón, 2019; Chirivella Caballero, 2010; Sosa Martínez, 2016).

Tabla No 4. Tipos de Mantenimientos.

\begin{tabular}{|c|c|}
\hline $\begin{array}{c}\text { Tipo de } \\
\text { mantenimiento }\end{array}$ & Descripción \\
\hline Correctivo & $\begin{array}{l}\text { Se realiza para identificar o corregir un problema, es decir, reparar cuando } \\
\text { algo falla. Consiste en actividades realizadas cuando se reacciona a una } \\
\text { interrupción de un sistema, subsistema, o parte de un equipo o servicio. }\end{array}$ \\
\hline Preventivo & $\begin{array}{l}\text { Consiste en evitar la ocurrencia de fallas en las máquinas o los equipos del } \\
\text { proceso. Este mantenimiento se basa en un plan, el cual contiene un } \\
\text { programa de actividades previamente establecido con el fin de anticiparse } \\
\text { a las anomalías. Existen varios tipos: mantenimiento programado } \\
\text { (intervalos fijos), mantenimiento de mejora, mantenimiento autónomo, } \\
\text { mantenimiento rutinario, mantenimiento periódico o sistemático. }\end{array}$ \\
\hline Predictivo & $\begin{array}{l}\text { Supone una inversión considerable en tecnología que permite conocer el } \\
\text { estado de funcionamiento de máquinas y equipos en operación, mediante } \\
\text { mediciones no destructivas. Las herramientas que se usan para el fin son } \\
\text { sofisticadas, por ello se consideran para maquinaría de alto costo, o que } \\
\text { forme parte de un proceso vital. }\end{array}$ \\
\hline $\begin{array}{l}\text { Productivo Total } \\
\text { (TPM) }\end{array}$ & $\begin{array}{l}\text { Es una estrategia compuesta por una serie de actividades ordenadas que } \\
\text { una vez implantadas ayudan a mejorar la competitividad de una } \\
\text { organización industrial o de servicios. Es considera como estrategia, ya que } \\
\text { ayuda a crear capacidades competitivas a través de la eliminación rigurosa } \\
\text { y sistemática de las deficiencias de los sistemas operativos. }\end{array}$ \\
\hline
\end{tabular}

\section{Fuente: Elaboración propia.}

La gestión del mantenimiento es responsable de conciliar los activos fijos, minimizar los tiempos de parada y los costos asociados a los mismos, por lo que una adecuada gestión de mantenimiento orientada hacia la calidad, apoya el incremento de la productividad (Rivero Rodriguez, 2016). La gestión moderna de mantenimiento debe encontrarse enfocada en reducir los costos globales del aparato productivo, en certificar el buen desempeño de equipos, en reducir al máximo el riesgo sobre las personas, también el riesgo en el medio ambiente; y crear todas las herramientas que sean necesarias para soportar estos objetivo (Monge Martínez, 2017).

En este sentido vale destacar que la correcta implementación del mantenimiento juega un rol importante si desea mantener las instalaciones, ofreciendo esta un buen servicio que 
garantice el confort de las personas que hacen uso de estas instalaciones. Como la infraestructura turística es la base tangible del desarrollo del turismo, se hace necesario una adecuada gestión de mantenimiento en cada infraestructura para que cada recurso turístico brinde lo mejor a cada visitante y sea de disfrute de cada uno de ellos (Hernández Santana, 2017), por lo que a continuación se aplica el procedimiento propuesto, con el fin de analizar, evaluar y proponer acciones correctivas para mejorar la gestión de mantenimiento de las infraestructuras para el desplazamiento de discapacitados en el Complejo Hotelero los Cactus Tuxpan, Varadero.

\section{Etapa 1. Preparación.}

\section{Paso 1.1. Caracterización de la entidad objeto de estudio:}

El Complejo Los Cactus Tuxpan con 4 estrellas, del Grupo Cubanacan y en contrato de administración con la cadena Be Live, desde junio 2018. Fue creado el 1 de Abril del 2018 como marca propia, son dos hoteles Cactus con 270 habitaciones y Tuxpan con 235, además 11 bares, 2 Restaurantes Buffet y 5 especializados, 2 piscina, jacuzzis, cancha de tenis, gimnasio, mini club y otros servicios, y que aunque está concebido como complejo hotelero la operación y los servicios son individuales, los clientes de un hotel no tienen acceso a ambos salvo casos excepcionales, Cactus opera con clientes adultos, Tuxpan opera como hotel de familia, incluye menores de edad.

Misión: Brindar servicios de hotelería para adultos y familias en un todo incluido, siempre con nuevos atributos gracias a la profesionalidad y valores humanos de nuestro personal, garantizando un trato amable y familiar en un ambiente de seguridad, confort e intimidad con la naturaleza.

Visión: Ser el mejor hotel Súper Todo Incluido para adultos y familias en el Caribe, preferido por la excelencia de sus servicios, un medio ambiente sustentable y altos valores de cubanía.

Los objetivos estratégicos de la entidad son:

$\checkmark$ Convertir en un baluarte invencible de los principios y la moral revolucionaria a los Cuadros y Trabajadores del Hotel.

$\checkmark$ Acometer las inversiones previstas para el mejoramiento del confort de nuestra planta hotelera.

$\checkmark$ Lograr la presencia de los más genuinos valores de nuestra identidad y de nuestra cultura en cada uno de los servicios de cara al cliente.

$\checkmark$ Crear una cultura de calidad en nuestros Directivos y Trabajadores que nos conlleve a la mejora continua de los procesos, reducir los ciclos de tiempo de los mismos y elevar la profesionalidad en los servicios.

$\checkmark$ Alcanzar un alto nivel de ambientación en todas las áreas del Hotel, que nos permita tener un Medio Ambiente verdaderamente sustentable.

$\checkmark$ Lograr el posicionamiento del Hotel en el mercado Internacional como el mejor Súper Todo Incluido del Caribe. 
Elevar paulatinamente la eficiencia económica en la operación del Hotel, para llegar a distinguirnos por nuestra alta rentabilidad.

\section{Paso 1.2. Conformación de equipo de trabajo:}

Se seleccionan un conjunto de 4 directivos de la organización y un especialista en la accesibilidad y movilidad ajeno a la instalación. En la tabla No 5 se muestra la aplicación del procedimiento.

Tabla 5. Nivel de competencia de los expertos seleccionados.

\begin{tabular}{|c|c|c|c|c|c|c|}
\hline $\begin{array}{l}\mathbf{N} \\
\mathbf{O}\end{array}$ & Nombre del experto & Cargo que ocupa & Kc & Ka & $\mathbf{K}$ & $\begin{array}{l}\text { Nivel de } \\
\text { competencia }\end{array}$ \\
\hline 1 & Dr. Rafael Gómez & $\begin{array}{l}\text { Especialista en } \\
\text { Accesibilidad y Movilidad } \\
\text { (Jefe de la Comisión). }\end{array}$ & 0.9 & 1.00 & 1 & Competente \\
\hline 2 & Ing. Raúl Santana & $\begin{array}{l}\text { Jefe de Servicios } \\
\text { Técnicos. }\end{array}$ & 1 & 0.82 & 0.9 & Competente \\
\hline 3 & $\begin{array}{l}\text { Esp. } \\
\text { Rodríguez }\end{array}$ & $\begin{array}{l}\text { Especialista de } \\
\text { mantenimiento a equipos } \\
\text { e instalaciones. }\end{array}$ & 0.9 & 0.94 & 0.9 & Competente \\
\hline 4 & Ms. Javier Valdés & Jefe de Mantenimiento. & 0.9 & 0.92 & 0.9 & Competente \\
\hline 5 & $\begin{array}{l}\text { Ing. } \\
\text { Cervantes }\end{array}$ & $\begin{array}{l}\text { Especialista A en } \\
\text { Seguridad y Salud en el } \\
\text { Trabajo (SSTT) para el } \\
\text { Turismo. }\end{array}$ & 1 & 0.78 & 0.9 & Competente \\
\hline
\end{tabular}

Fuente: Elaboración propia.

El equipo de trabajo quedó conformado por:

$\checkmark$ Especialista en Accesibilidad y Movilidad (Jefe de la Comisión).

$\checkmark$ Jefe de Servicios Técnicos.

$\checkmark$ Jefe de Mantenimiento.

$\checkmark$ Especialista de mantenimiento a equipos e instalaciones.

$\checkmark$ Especialista A en Seguridad y Salud en el Trabajo (SSTT) para el Turismo.

Etapa 2. Realización del diagnóstico de la situación existente en la gestión del mantenimiento en El Complejo Los Cactus Tuxpan, Varadero.

\section{Paso 2.1. Preparación del plan de aspectos a diagnosticar.}

El jefe de la comisión prepara el plan a desarrollar, el cual tiene como objetivo general: determinar los problemas que presentan la gestión de mantenimiento orientado al desplazamiento de discapacitados en El Complejo Los Cactus Tuxpan. Se analiza la situación de las áreas de Recepción, Recreación, Habitaciones y Gastronomía (Bares y Restaurant). Esta inspección fue realizada en los meses de julio y agosto del 2019 con vista de una mejor preparación de la institución para el alza turística en los meses venideros. El plan tiene en cuenta organización, planificación, ejecución y control de los mantenimientos programados que realiza la institución orientado a mejorar el desplazamiento de personas con alguna limitación o discapacidad. Se analizarán los mantenimientos preventivos, correctivos y predictivos. 
Paso 2.2. Asignación de tareas. La tabla 6 muestra la relación de las tareas y su supervisor.

Tabla 6. Asignación de las tareas

\begin{tabular}{ll}
\hline Miembro de la Comisión & Área \\
\hline Jefe de Mantenimiento & Recreación \\
Especialista de mantenimiento a equipos e instalaciones. & Recepción \\
Especialista A en SSTT para el Turismo. & Habitaciones \\
Jefe de Servicios Técnicos & Gastronomía \\
\hline
\end{tabular}

Fuente: Elaboración propia.

Etapa 3. Determinación de los principales problemas que afectan la gestión del mantenimiento en El Complejo Los Cactus Tuxpan.

\section{Paso 3.1. Problemas detectados en la gestión de mantenimiento:}

A partir del diagnóstico de la situación existente se determina que la gestión mantenimiento de las infraestructuras orientadas al desplazamiento de personas con discapacidades es deficiente, los principales problemas detectados son:

1. Mal estado técnico las infraestructuras destinas a discapacitados (elevadores rotos o con desperfectos técnicos, deterioro de las señalizaciones y baños públicos, barandas falta de pintura, aceras y rampas con alto grado de deterioro, pisos resbaladizos.

2. El personal que posee la empresa para la ejecución del mantenimiento no está altamente calificado, lo que trae consigo que en ocasiones se presentan encarecimientos del trabajo al utilizar más recursos que los necesarios.

3. No existe un adecuado control de la ejecución de las tareas, por lo que en muchos casos existen interferencias y desorganizaciones que disminuyen la productividad del trabajo e influyen en el incumplimiento de las fechas de terminación de los trabajos de mantenimiento.

Etapa 4. Propuestas de soluciones a los problemas detectados. La comisión encargada de la inspección se reúne con todas las personas involucradas en la entidad con la gestión del mantenimiento, se analizan los problemas detectados y a partir de una tormenta de ideas se recogen las soluciones propuestas.

Paso 4. 1. Listado de soluciones. Para dar solución a los problemas detectados en El Complejo Hotelero los Cactus Tuxpan relacionados con la gestión del mantenimiento. Se proponen las medidas soluciones (Tabla 7). 
Tabla 7. Propuesta de medidas correctivas.

\begin{tabular}{|c|c|c|c|}
\hline Problemas & Medidas correctivas & $\begin{array}{l}\text { Fecha } \\
\text { ejecución }\end{array}$ & Responsable \\
\hline $\begin{array}{l}\text { 1. Mal estado } \\
\text { técnico las } \\
\text { infraestructuras } \\
\text { destinas a } \\
\text { discapacitados. }\end{array}$ & $\begin{array}{l}\text { Programar } \\
\text { mantenimientos } \\
\text { preventivos con la } \\
\text { documentación } \\
\text { necesaria } \\
\text { correspondencia en } \\
\text { las necesidades de la } \\
\text { institución para el } \\
\begin{array}{l}\text { desarrollo de las } \\
\text { actividades. }\end{array}\end{array}$ & Mensual & $\begin{array}{l}\text { Jefe de } \\
\text { Mantenimiento }\end{array}$ \\
\hline & $\begin{array}{l}\text { Incluir en el } \\
\text { presupuesto anual al } \\
\text { mantenimiento de la } \\
\text { infraestructura dirigida a } \\
\text { los discapacitados. }\end{array}$ & Anual & $\begin{array}{ll}\text { Jefe } & \text { de } \\
\text { Inversiones } & \end{array}$ \\
\hline $\begin{array}{l}\text { 2. El personal para } \\
\text { la ejecución del } \\
\text { mantenimiento } \\
\text { no está } \\
\text { altamente } \\
\text { calificado. }\end{array}$ & $\begin{array}{l}\text { Desarrollar programas } \\
\text { de capacitación } \\
\text { dirigidos al personal } \\
\text { encargado r del } \\
\text { mantenimiento. }\end{array}$ & Trimestral & $\begin{array}{l}\text { Director } \\
\text { Recursos } \\
\text { Humanos }\end{array}$ \\
\hline $\begin{array}{l}\text { 3. No existe un } \\
\text { adecuado } \\
\text { control de la } \\
\text { ejecución de las } \\
\text { tareas }\end{array}$ & $\begin{array}{l}\text { Verificar } \\
\text { cumplimiento de los } \\
\text { planes de las } \\
\text { actividades propuestas } \\
\text { para el mantenimiento. } \\
\text { Controlar los recursos } \\
\text { destinados a cada } \\
\text { actividad. }\end{array}$ & Sistemáticamente & $\begin{array}{l}\text { Jefe de } \\
\text { Mantenimiento }\end{array}$ \\
\hline
\end{tabular}

Fuente: Elaboración propia.

\section{Paso 4.2. Propuesta de un plan de Mantenimiento.}

Se aplica el Método Pareto (Tabla 8) con el objetivo de conocer el orden de prioridad que se debe tener para ejecutar el mantenimiento.

Tabla 8. Método Pareto.

\begin{tabular}{lllll}
\hline Infraestructuras & $\begin{array}{l}\text { (Quejas) EE } \\
\text { (efecto económico) }\end{array}$ & $\%$ & $\begin{array}{l}\text { EE } \\
\text { acumulado }\end{array}$ & $\begin{array}{l}\% \\
\text { acumulado }\end{array}$ \\
\hline Elevadores & 94 & 78.33 & 94 & 78.33 \\
Rampas & 10 & 8.33 & 104 & 86.66 \\
Aceras & 6 & 5.00 & 110 & 91.66 \\
Pisos & 5 & 4.17 & 115 & 91.66 \\
Señalización & 3 & 2.50 & 118 & 95.83 \\
Barandas & 2 & 1.67 & 120 & 98.33 \\
Total & 120 & 100 & & 100 \\
\hline
\end{tabular}

Fuente: Elaboración propia.

Luego de aplicar el Método Pareto y determinar que la infraestructura que debe ser priorizada en el mantenimiento son los elevadores porque representa el $78.33 \%$ (el 
porcentaje más cercano a 80) de las quejas presentadas por clientes durante los meses inspeccionados, se le propone el siguiente plan de mantenimiento preventivo (Tabla 9) bajo el concepto de que es preferible invertir en las revisiones que sufrir una avería más profunda cuya reparación sea más costosa y requiera de la paralización de la máquina durante un largo tiempo y genere molestias en los turistas.

Tabla 9. Propuesta de un plan preventivo para los elevadores.

\begin{tabular}{|c|c|c|c|c|}
\hline $\begin{array}{l}\text { Área: } \\
\text { Recepción- } \\
\text { Alojamiento }\end{array}$ & $\begin{array}{l}\text { Responsable: } \\
\text { Jefe de } \\
\text { Mantenimiento }\end{array}$ & $\begin{array}{l}\text { Tipo de } \\
\text { mantenimiento: } \\
\text { Preventivo }\end{array}$ & $\begin{array}{l}\text { Equipos sometidos } \\
\text { al Mantenimiento: } \\
\text { Elevadores }\end{array}$ & $\begin{array}{l}\text { Lugar: } \\
\text { Hotel } \\
\text { Tuxpan. }\end{array}$ \\
\hline
\end{tabular}

Descripción del mantenimiento mensual. Se deben hacer revisiones rutinarias, sustituir piezas desgastadas, hacer la inspección del ascensor correspondiente, implementar las modificaciones que obliguen las normas y leyes que exija la administración pública. Comprobar los componentes de cabina, la alarma, verificar el arranque, la parada, la nivelación, revisar la apertura, y cierre de puertas de la cabina y asegurarse del funcionamiento y señalización de las puertas de los pisos.

Descripción del mantenimiento trimestralmente. Se debe limpiar el foso, revisar el freno, controlar el nivel de aceite de motores, máquina y posibles fugas, limpiar la pisadera de puertas de cabina y limpiar el cuarto de máquinas.

Descripción semestral del mantenimiento. Se deben realizar tareas de control sobre los siguientes elementos: luz de emergencia, limpieza y revisión de las puertas de la cabina, operador y controlar el estado de patinaje y tensión de cables.

Descripción del mantenimiento anual. Se debe revisar el estado de amarres cabina y contrapeso, verificar paracaídas y articulaciones, polea, impulsores y detectores, fugas de pistón, mangueras y estado de retenes y tuberías, comprobar renivelación, limpieza de techo y bajos de la cabina.

Herramientas Necesarias:

Escalera mecánica, aceras móviles, montacargas, herramientas manuales, dispositivos de pruebas (amperímetro y voltímetro).

Plan de Frente a una emergencia lo primero que se debe hacer es conservar la calma.

Seguridad En caso de bloqueo, el ascensor cuenta con una batería para la luz de frente a emergencia, alarma sonora y citófonos intercomunicados con recepción. En imprevistos caso de incendio o de otro tipo de emergencia no se deben usar los elevadores. Al ingresar o salir del elevador revisar si está al nivel del suelo. No es recomendable realizar transporte de cargas pesadas que excedan el peso máximo recomendado.

Fuente: Elaboración propia.

El mantenimiento como se evidencia en esta investigación, representa un arma en la seguridad laboral y permite la detección de fallas producidas por el desgaste de piezas, lo que permite una adecuada programación en el cambio o reparación de las mismas y con esto disminuye los costos provocados por paradas del proceso, las molestias ocasionadas a los clientes y maximiza el beneficio global.

\section{Conclusiones.}

- De la revisión realizada se observa que Cuba es un destino de gran demanda de personas con discapacidad, por tal motivo sus instalaciones deben contar con los 
estándares para que cualquier individuo puede recrearse o hacer uso de los servicios en cualquier instalación sin importar su condición física.

- El mantenimiento de infraestructuras para el desplazamiento de discapacitados simboliza una mejora continua de las instituciones hoteleras; mediante el incremento de la disponibilidad, efectividad y confiabilidad, por lo que de esta forma se aumenta la producción y se maximiza el beneficio global del turismo.

- Se desarrolla un mapa del estudio bibliométrico del término "desplazamientos de discapacitados" en base a la co-ocurrencia de palabras que demuestran que las infraestructuras adecuadas, lugares accesibles y los desplazamientos para personas discapacitadas constituyen referentes de estudios a nivel mundial según la base de datos Sciencie Direct.

- A partir del procedimiento propuesto se detectaron los principales problemas que afectan la gestión de mantenimiento de las infraestructuras para el desplazamiento de discapacitados en el Complejo Hotelero los Cactus Tuxpan, Varadero.

- Se propone un plan de medidas correctivas con el fin de erradicar los problemas detectados durante el diagnóstico para lograr una adecuada gestión del mantenimiento de las infraestructuras destinadas a discapacitados en la entidad objeto de estudio.

\section{Referencias bibliográficas.}

Agovino, M., Casaccia, M., Garofalo, A., \& Marchesano, K. (2017). Tourism and disability in Italy. Limits and opportunities. Tourism management perspectives, 23, 58-67.

Alonso Gatell, A., \& Leyva Fontes, C. (2008). Paseos peatonales costeros. Gestión de sus Recursos. Rev Invest. Mar,29(1), 63-72

Artola Pimentel, M. d. L. (2002). Modelo de evaluación del desempeño de empresas perfeccionadas en el tránsito hacia empresas de clase en el sector de servicios ingenieros de Cuba. (Tesis de Doctorado), Universidad de Matanzas "Camilo Cienfuegos", Cuba.

Barba, S. E. (2018). Diseño de una ruta turística accesible e inclusiva, para turistas potenciales y con discapacidad física leve de movilidad limitada, en el cantón Francisco de Orellana. (Tesis de licenciatura), Universidad Central del Ecuador. http://www.dspace.uce.edu.ec/handle/25000/15054

Borroto Pentón, Y. (2005). Contribución al mejoramiento de la gestión del mantenimiento en hospitales en Cuba. Aplicación en la provincia Villa Clara. (Tesis de Doctorado), Universidad Central "Marta Abreu" de Las Villas, Cuba.https://dspace.uclv.edu.cu/handle/123456789/3562 
Buitrago Falcón, D. (2019). Ampliación y desarrollo de un Plan de Mantenimiento Predictivo para el análisis del estado de los motores asíncronos. (Tesis de máster), Universitat Politècnica de València, España.http://hdl.handle.net/10251/126442

Carossia, E. d. L., Narvaez, E. L., \& Peluc, M. H. (2007). Turismo y desarrollo: La accesibilidad hotelera como indicador de calidad de la oferta. https://scholar.google.es/scholar?as_q=discapacitados\&as_epq=\&as_oq=\&as_eq $=\& a s \_o c c t=a n y \& a s \_s a u t h o r s=C a r o s s i a \& a s \_p u b l i c a t i o n=\& a s \_y l o=\& a s \_y h i=\& h$ $1=$ es\&as_sdt=0\%2C5.

Castillo García, M. (2019). El rol "accesible" del espacio público. Revista Semestral Arquis., 8(1).

Castro-Alfaro, A., Paz-Marcano, E., \& Pinto-Aragón. (2019). Administración de los protocolos y accesibilidad para personas con discapacidad. Caso Hotel Caribe en la ciudad de Cartagena de Indias. Revista Vínculos: Ciencia Tecnología y Sociedad, 16(2), 350-362. doi:https://doi.org/10.14483/2322939X.16575

Castro, J. (2017). Las facilidades turísticas del sector hotelero, y su contribución al turismo inclusivo del cantón Ambato provincia de Tungurahua Universidad Técnica de Ambato.https://repositorio.uta.edu.ec/hand-le/123456789/25181

Chirivella Caballero, M. (2010). La Gestión del Mantenimiento Hotelero y la Eficiencia. Revista de Ingeniería del Mantenimiento en Canarias 1, 91-93.

Clemente Soler, J. A., Bote Díaz, M., \& Sánchez Vera, P. (2018). El turismo social accesible como nuevo modelo turístico. Cuadernos de turismo (41). doi:https://doi.org/10.6018/turismo.41.326981

Cruz, G. (2019). Perspectivas teóricas para el abordaje de los movimientos sociales y su incorporación en el campo de la investigación en turismo. Turismo y Sociedad, 25, 195-216. doi:https://doi.org/10.18601/01207555.n25.10

Domínguez, T., Fraiz, J. A., \& Alén, M. E. (2016). Discapacidad y alojamientos turísticos en España. Revisata de Turismo y Patrimonio Cultural, Vol. 13 (N.o 4 Special Issue), 771-787.

Domínguez Vila, T., Fraiz Brea, J. A., \& Alén González, M. E. (2011). Turismo y accesibilidad. Una visión global sobre la situación de España. Cuadernos de turismo (28), 23-45.

Fraiz Brea, J. A., Alén González, E., \& Domínguez Vila, T. (2008). La accesibilidad como oportunidad de mercado en el management de destinos turísticos. Revistas de Análisis Turísticas, 5(1), 30-45. 
Gómez Bañuelos, D., Perez Gaxiola, A., \& Woolfolk Gallego, L. E. (2018). Turismo accesible en la industria restaurantera de Bahia de Kino, Sonora. . Turismo y Desarrollo, 11(24).

Gómez Bañuelos, D., Pérez Gaxiola, A., \& Woolfolk Gallego, L. E. (2018). Turismo accesible en la industria restaurantera de Bahia de Kino, Sonora. Revista Turismo y Desarrollo local sostenible No. 24.

González Echavarría, J. A., Martínez Delgado, E., Barreto San Germán, E. L., Espinosa Alfonso, V. M., \& Cabrera Gómez, J. (2020). Modelo con enfoque logístico para diagnosticar la gestión de mantenimiento de una entidad productora de envases. Ingeniería Mecánica, 23(2).

Guerrero, P. (2018). ¿Cómo medir la accesibilidad turística? Importancia de los sistemas de indicadores para validar destinos turísticos accesibles. . Tierra Infinita, Vol. 4(1), 106-118. doi: https://doi.org/10.32645/26028131.785

Haro Ayala, J. E. (2018). La Gestion de Mantenimiento Industrial y la productividad en una empresa de alimentos, Callao 2017. (Tesis de grado), Universidad César Vallejo.https://hdl.handle.net/20.500.12692/23261

Hernández Santana, J. A. (2017). Estudio del sistema de mantenimiento en las pasarelas en el Hotel Playa Cayo Santa María. (Tesis de grado), Universidad Central" Marta Abreu" de Las Villas. , Cuba. http://dspace.uclv.edu.cu:8089/handle/123456789/8494

Jiménez-Castro, M. A. (2017). Modelo de gestión de mantenimiento para el Hotel Papagayo Secrets Resorts \& Spa. Tecnológico de Costa Rica, Costa Rica. http://hdl.handle.net/2238/9624

Jurado Almonte, J. M. (2014). El turismo accesible en Andalucía y Portugal. Cuadernos de turismo (33), 121-150.

Kastenholz, E. (2009). Turismo accesible como ejemplo de responsabilidad social en las empresas y destinos turísticos. el caso de Lousã (Portugal). Rotur/Revista de Ocio Y Turismo $\mathrm{N}^{\circ}$ 2, 175 - 194.

Kastenholz, E., Eusébio, C., Figueiredo, E., \& Lima, J. (2012). Accessibility as competitive advantage of a tourism destination: The case of Lousã Field guide to case study research in tourism, hospitality and leisure: Emerald Group Publishing Limited.

Martínez Carrillo, M. J., \& Boujrouf, S. (2020). Turismo accesible para todos. Evaluación del grado de accesibilidad universal de los parques y jardines de Marrakech. PASOS Revista de Turismo y Patrimonio Cultural, 18(1), 57-81. doi:https://doi.org/10.25145/j.pasos.2020.18.004 
Mercado Britton, D., \& Villorina Seña, J. (2010). Mantenimiento hotelero en Colombia revisión del estado actual y propuesta de valor. (Tesis de grado), Universidad EAFIT, Medellín, Colombia. http://hdl.handle.net/10784/428

Miranda, H. (2017). Deportes extremos para discapacitados en base al turismo accesible en la ciudad de Baños de Agua Santa (Tesis de Pregrado), Universidad Técnica de Ambato. https://repositorio.uta.edu.ec/handle/123456789/25143

Monge Martínez, H. Y. (2017). Diseño de un modelo de gestión de mantenimiento para el departamento de Ingeniería del hotel San José Costa Rica Marriott. (Tesis de grado), Instituto Tecnológico de Costa Rica., Costa Rica. http://hdl.handle.net/2238/9637

Negrín Sosa, E. (2003). El mejoramiento de la administración de operaciones en empresas de servicios hoteleros. (Tesis de Doctorado), Universidad de Matanzas, Matanzas, Cuba.

Nonome, E. (2019). El neuroturismo y su relación con el turismo accesible como una propuesta para la ciudad de Lima. (Tesis de doctorado), Universidad de San Martín de Porres, Lima, Perú.http://www.repositorioacademico.usmp.edu.pe/handle/usmp/5294

Organización de las Naciones Unidas (ONU) (2014). Convención sobre los derechos de las personas con discapacidad. Guía de información.[En línea] Disponible en https://www.ohchr.org/Documents/publications/CRPD_TrainingGuide_PTS19_ sp.pdf, [11 de diciembre de 2015].

Pinotti, M. M. (2018). La accesibilidad de los alojamientos hoteleros en la ciudad de Buenos Aires. . (Tesis de Maestría), Universidad de Buenos Aires Buenos Aires., Argentina.

Proaño Cárdenas, C. I. (2018). Propuesta de metodología de implementación de un sistema de gestión de mantenimiento centrado en la confiabilidad (MCC), basado en las normas ISO 14224, SAE JA1011 y SAE JA1012, en la central hidroeléctrica Coca Codo Sinclair-CELEC EP. (Tesis de Maestria), Escuela Politécnica Nacional, Quito. http://bibdigital.epn.edu.ec/handle/15000/19787

Rifaï, T. (2014). Turismo internacional y agilización de visados. Estudios Turísticos(200), 9-18.

Rivero Rodriguez, L. D. (2016). Herramienta informática para la gestión de mantenimiento en el hotel "Meliá Las Dunas". (Tesis de grado), Universidad Central "Marta Abreu" de Las Villas. Cuba.http://dspace.uclv.edu.cu:8089/handle/123456789/7269 
Sanz Labrador, M. Y. (2018). La inclusión de las personas con discapacidad a las actividades físico-recreativas turísticas en Villa Clara. Ciencia y Actividad Física, $5(2), 36-47$.

Silveira, Y., Silveira, R., \& Castellanos, G. (2010). Impacto social y económico de la industria turística cubana. Revisata de Turismo y Desarrollo,3(8).

Sosa Martínez, D. A. (2016). Selección del tipo de mantenimiento a aplicar en los sistemas tecnológicos y equipos del Kurhotel" Escambray". (Tesis de grado), Universidad Central "Marta Abreu" de Las Villas. , Cuba. http://dspace.uclv.edu.cu:8089/handle/123456789/7285

Suárez Falcón, H., Verano Tacoronte, D., \& García Santana, A. (2016). La movilidad urbana sostenible y su incidencia en el desarrollo turístico. Gestión y ambiente, 19(1), 48-62.

Suárez Fragas, Y., Medina Peña, D., \& Hernández Alfonso, P. M. (2016). Sistema automatizado para la gestión del mantenimiento de equipos (módulos administración y solicitud de servicio). Revista Ciencias Técnicas Agropecuarias, 24(5), 85-90.

Tite, G., Carrillo, D., \& Ochoa, M. (2021). Turismo accesible: estudio bibliométrico. . Turismo y Sociedad, Vol. XXVIII, 115-132.doi: https://doi.org/10.18601/01207555.n28.06

Vergara Peña, E. E. (2016). Propuesta de implementación de playas accesibles para personas con discapacidad motriz y movilidad reducida en el cantón Salinas, Provincia de Santa Elena. (Tesis de Diploma), Universidad Católica de Santiago de Guayaquil.

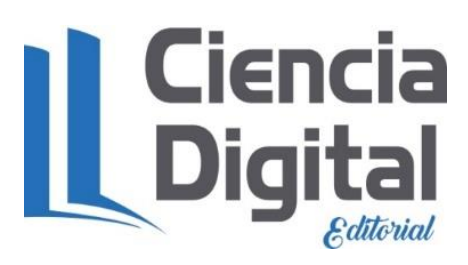




\section{PARA CITAR EL ARTÍCULO INDEXADO.}

Sangroni Laguardia, N., Pérez Castañeira, J. A., Alba Cruz, R., Jiménez Valero, B., \& Velastegui López, L. E. (2021). Análisis de la Gestión del Mantenimiento orientado a infraestructuras para el desplazamiento de discapacitados en el Complejo Hotelero los Cactus Tuxpan, Varadero. ConcienciaDigital, 4(2.1), 131-153. https://doi.org/10.33262/concienciadigital.v4i2.1.1716

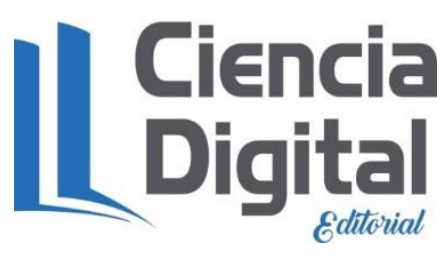

El artículo que se publica es de exclusiva responsabilidad de los autores y no necesariamente reflejan el pensamiento de la Revista Conciencia Digital.

El artículo queda en propiedad de la revista y, por tanto, su publicación parcial y/o total en otro medio tiene que ser autorizado por el director de la Revista Conciencia Digital.

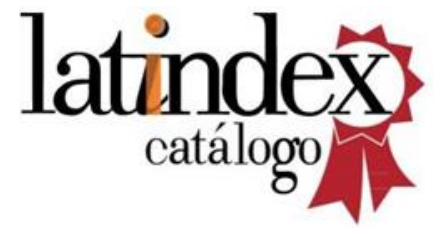

